\title{
Insulin Resistance and Hyperinsulinemia in Individuals with Small, Dense, Low Density Lipoprotein Particles
}

Gerald M. Reaven, Y.-D. Ida Chen, Jorgen Jeppesen, Pierre Maheux, and Ronald M. Krauss *

Department of Medicine, Stanford University School of Medicine and Geriatric Research Education and Clinical Center, Department of Veterans Affairs Medical Center, Palo Alto, California 94304; and * Donner Laboratory, Lawrence, University of California, Berkeley, California 94720

\section{Abstract}

Subjects characterized by a predominance of small LDL particles ( pattern B) have changes in plasma triglyceride (TG) and HDL-cholesterol concentrations consistent with the presence of resistance to insulin-mediated glucose uptake. To pursue this issue, plasma glucose and insulin responses to oral glucose, insulin-mediated glucose disposal, and lipoprotein concentrations were measured in subjects categorized on the basis of LDL peak diameter measured by gradient gel electrophoresis. Subjects with pattern $B$ had higher $(P<0.05-0.001)$ total integrated plasma glucose $(20.7 \pm 1.0 \mathrm{mmol} /$ liter $\cdot h)$ and insulin $(1,743 \pm 293 \mathrm{pmol} /$ liter $\cdot h)$ responses to oral glucose compared with glucose $(16.3 \pm 0.4$ and $19.2 \pm 0.8 \mathrm{mmol} / \mathrm{liter} \cdot \mathrm{h})$ and insulin $(856 \pm 60$ and $1,222 \pm 168 \mathrm{pmol} /$ liter $\cdot h)$ responses in those with either pattern $A$ or an intermediate pattern. Pattern B individuals were shown to be more insulin resistant on the basis of higher steady state plasma glucose concentrations (SSPG, 10.4 $\pm 1.0, P<0.002$, vs. $7.5 \pm 0.7$ and $6.0 \pm 0.4 \mathrm{mmol} /$ liter) after a constant infusion of somatostatin, glucose, and insulin than those with either the intermediate or pattern $A$ subclass. Pattern B subjects also had higher concentrations of $(P<0.001)$ TG $(1.98 \pm 0.15$ vs. $1.33 \pm 0.17$ and $0.77 \pm 0.05$ mmol/liter $)$ and lower $(P<0.01-0.001)$ HDL cholesterol $(1.12 \pm 0.06$ vs. $1.34 \pm 0.05$ vs. $1.45 \pm 0.05 \mathrm{mmol} /$ liter $)$ than those with either the intermediate or pattern A. Finally, significant $(P<0.001)$ correlation coefficients existed between LDL diameter and SSPG $(r=-0.44)$; glucose $(r=-0.41)$ and insulin $(r=-0.38)$ responses; TG $(r=-0.65)$ and HDL-cholesterol $(r=0.42)$ concentrations; and systolic $(r=-0.34)$ and diastolic $(r=-0.34)$ blood pressure. Thus, pattern B subjects are insulin resistant, have higher glucose, insulin, and TG, lower HDL-cholesterol levels, and higher blood pressure than those with pattern A or intermediate. (J. Clin. Invest. 1993. 92:141-146.) Key words: LDL particle diameter $\cdot$ glucose and insulin responses - resistance to insulin-mediated glucose uptake $\cdot$ lipid and lipoprotein concentrations $\cdot$ blood pressure $\bullet$ pattern B LDL

\section{Introduction}

Although heterogeneity in size and density of LDL particles has been appreciated for some time (1-3), results of more re-

Address reprint requests to Gerald M. Reaven, M.D., GRECC (182B), VA Medical Center, 3801 Miranda Avenue, Palo Alto, CA 94304. Received for publication 3 December 1992 and in revised form 5 February 1993.

The Journal of Clinical Investigation, Inc.

Volume 92, July 1993, 141-146 cent studies have begun to define the clinical relevance of these earlier observations. There is now substantial evidence (4-6) indicating that individuals with smaller LDL particles are at increased risk to develop coronary heart disease. Analysis of LDL particle size distributions by nondenaturing gradient gel electrophoresis has identified multiple, distinct LDL subclasses and has shown that LDL in most individuals can be characterized by a predominance of larger LDL (diameter usually larger than $255 \AA$, subclass pattern A) or smaller LDL ( $\leq 255 \AA$, subclass pattern $B$ ). Individuals with subclass pattern $B$ have been shown to have higher plasma triglyceride (TG) ${ }^{1}$ and lower HDL-cholesterol concentrations $(5,6)$. Similar changes in plasma TG and HDL-cholesterol concentrations have also been shown to be associated with resistance to insulin-mediated glucose uptake and/or hyperinsulinemia (7-11). Given these observations, it seemed reasonable to raise the possibility that subclass pattern B may also be associated with insulin resistance. The study to be presented was initiated to address this hypothesis and was performed in 100 nondiabetic and healthy individuals.

\section{Methods}

The study population consisted of 100 normal adults, 52 women and 48 men, who responded to a newspaper advertisement describing our experimental goal. Their mean age $( \pm S D)$ was $44 \pm 13$ yr (range: $20-71$ $\mathrm{yr}$ ), and their mean ( $\pm \mathrm{SD}$ ) body mass index (BMI) was $24.8 \pm 3.6 \mathrm{~kg} /$ $\mathrm{m}^{2}$ (range: $18.0-34.5 \mathrm{~kg} / \mathrm{m}^{2}$ ). They had no history or symptoms of any known disease and had a normal physical examination and routine findings on general blood chemical analyses, hemogram, and resting electrocardiogram. Subjects were not receiving any medicine known to influence lipid metabolism or glucose tolerance. After subjects gave their informed written consent, they were admitted to the Stanford University General Clinical Research Center, and the following tests were performed.

Arterial blood pressure was measured with a mercury sphygmomanometer, using the disappearance of Korotkoff sounds (phase V) as criterion for the determination of diastolic blood pressure. The reported blood pressures are the arithmetic means of measurements made on three separate days before venipuncture and after subjects sat for $\geq 5 \mathrm{~min}$ in a quiet environment.

Venous blood was drawn on two occasions after an overnight fast for measurement of plasma TG (12), cholesterol (13), and for separation of various lipoprotein fractions (14). Plasma glucose (15) and insulin (16) concentrations were measured after an overnight fast before and 30,60,120, and $180 \mathrm{~min}$ after a 75 -g oral glucose load. Finally, insulin-mediated glucose uptake was estimated by a modification of the insulin suppression test (17) originally described by our research

1. Abbreviations used in this paper: BMI, body mass index; SSPG, steady state plasma glucose; SSPI, steady state plasma insulin; TG, triglycerides. 
group (18). This study was also performed after an overnight fast and involved the continuous intravenous infusion for $180 \mathrm{~min}$ of somatostatin $(5 \mu \mathrm{g} / \mathrm{min})$, insulin $\left(25 \mathrm{mU} / \mathrm{m}^{2} \cdot \min \right)$, and glucose $(240 \mathrm{mg} /$ $\mathrm{m}^{2} \cdot \mathrm{min}$ ) into an indwelling teflon catheter in a superficial antecubital vein. Venous blood samples were obtained from a similar catheter inserted in a contralateral antecubital vein and kept patent by a slow infusion of $0.9 \%$ sodium chloride. Blood was obtained every $10 \mathrm{~min}$ during the last $30 \mathrm{~min}$ for measurement of plasma glucose and insulin concentrations, and the mean value of these four measurements was used to calculate the steady state plasma insulin (SSPI) and steady state plasma glucose (SSPG) concentrations. Under these experimental circumstances, the SSPI was comparable in all individuals and the SSPG concentration provided a measure of insulin-mediated glucose disposal; the higher the SSPG, the more insulin-resistant the individual.

An aliquot of venous blood drawn after an overnight fast from each individual was also analyzed for size distribution of LDL particles by gradient gel electrophoresis (19). Subjects were classified into three groups on the basis of distinct LDL subclass patterns (5): pattern A was defined as an LDL subclass pattern with the major gradient gel peak at a particle diameter of $\geq 255 \AA$ and the presence of a minor peak of smaller LDL particles. Pattern $B$ had the major peak at a particle diameter of $<255 \AA$, with skewing of the curve toward larger particle diameters. Subjects with some of the characteristics of both patterns A and B was defined as intermediate pattern. The mean peak particle diameters for $A$, intermediate, and B patterns were 268,261 , and $250 \AA$, respectively ( see Table I).

Statistical analysis. Two-way analysis of variance was used for comparison of data collected over multiple time points among the three groups. Multiple samples obtained at a single time point, such as a fasting state, were compared by one-way analysis of variance (20).

Pearson correlation coefficients (21) were calculated to determine the relationship between LDL diameter and various measures of insulin resistance and lipid values. Partial correlation coefficients (21), adjusting for designated variables, were used to ascertain the direct relationship between LDL diameter and other variables (see results for specific adjustments). Since some of the metabolic variables do not show normal distributions, (TG, insulin response, and SSPG), Spearman correlation was applied in these situations (22). Finally, multiple regression analysis was performed using different models (see Results), consisting of different independent variables, with the dependent variable being LDL diameter.

All results were presented as mean $\pm \mathrm{SEM}$, with the exception of Tables I and IV, in which the basic demographic data were given as mean \pm SD.

Table I. Baseline Characteristics of Subjects Divided into Pattern $A$, an Intermediate Pattern, or Pattern B on the Basis of Their LDL Particle Diameter*

\begin{tabular}{|c|c|c|c|}
\hline Variable & $\begin{array}{c}\mathrm{A} \\
(n=52)\end{array}$ & $\begin{array}{c}\text { INT } \\
(n=29)\end{array}$ & $\begin{array}{c}\text { B } \\
(n=19)\end{array}$ \\
\hline LDL diameter $(\AA)$ & $268 \pm 4$ & $261 \pm 3^{\ddagger}$ & $250 \pm 4^{811}$ \\
\hline Age $(y r)$ & $40 \pm 13$ & $48 \pm 13^{\ddagger}$ & $45 \pm 11$ \\
\hline $\mathrm{BMI}\left(\mathrm{kg} / \mathrm{m}^{2}\right)$ & $24.0 \pm 3.4$ & $25.1 \pm 3.3$ & $26.5 \pm 3.9^{5}$ \\
\hline Gender (males/females) & $20 / 32$ & $16 / 13$ & $12 / 7^{\S}$ \\
\hline Systolic blood pressure $(\mathrm{mmHg})$ & $114 \pm 11$ & $119 \pm 15$ & $127 \pm 18^{8 \mid}$ \\
\hline Diastolic blood pressure $(\mathrm{mmHg})$ & $72 \pm 8$ & $73 \pm 10$ & $81 \pm 10^{8 \mid}$ \\
\hline
\end{tabular}

\footnotetext{
* Mean \pm SD. INT, intermediate. ${ }^{\ddagger}$ INT pattern significantly different $(P<0.05)$ than pattern A. ${ }^{\S}$ Pattern B significantly different $(P$ $<0.05)$ than pattern A. "Pattern B significantly different $(P<0.05)$ than INT pattern.
}
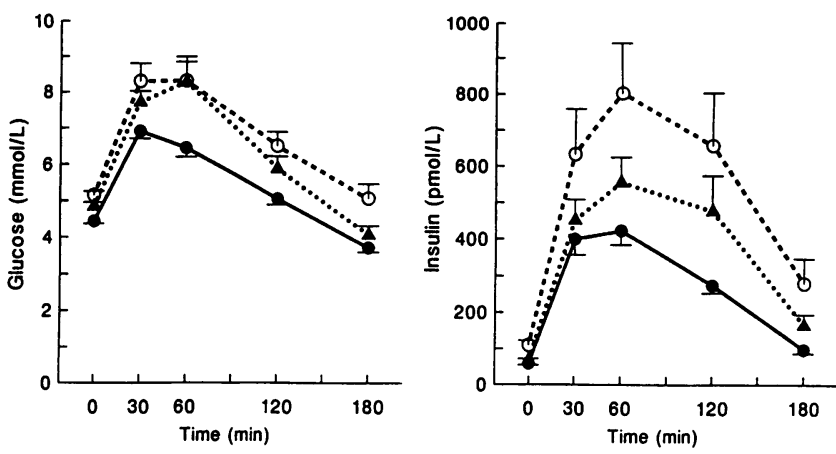

Figure 1. Plasma glucose (left) and insulin (right) responses to a 75-g oral glucose challenge in subjects divides into pattern $A(\bullet-\bullet)$, an intermediate pattern $(\Delta \cdots \Delta)$, and pattern $B(0 \cdots 0)$ on the basis of their LDL particle diameter.

\section{Results}

On the basis of LDL particle size, the population consisted of 52 subjects with pattern A, 29 subjects with the intermediate pattern, and 19 with pattern B. The average LDL diameters of the three groups are given in Table I, and all three groups were significantly different from each other. Baseline characteristics of the three groups in Table I demonstrate that individuals with pattern B consisted of more male individuals and had a higher BMI and higher systolic and diastolic blood pressures than subjects with either pattern A or the intermediate pattern.

Plasma glucose and insulin responses to oral glucose challenge in the three groups are shown in Fig. 1, and these data demonstrate that pattern $B$ individuals were relatively glucose intolerant $(P<0.05-0.0005)$ and hyperinsulinemic $(P<0.01-$ 0.0005 ) as compared with the other two groups. The plasma glucose and insulin responses of those with the intermediate pattern were higher than in those with pattern $A$ and lower than in those with pattern B and were significantly different $(P$ $=0.05-0.005)$ from both. The SSPG and SSPI concentrations of the three groups are illustrated in Fig. 2. SSPG concentrations (Fig. 2, right) were significantly higher ( $10.4 \pm 1.0 \mathrm{mmol} /$ liter, $P<0.05-0.001)$ in pattern B subjects compared those with pattern $A(6.0 \pm 0.4 \mathrm{mmol} /$ liter $)$ or the intermediate pat-
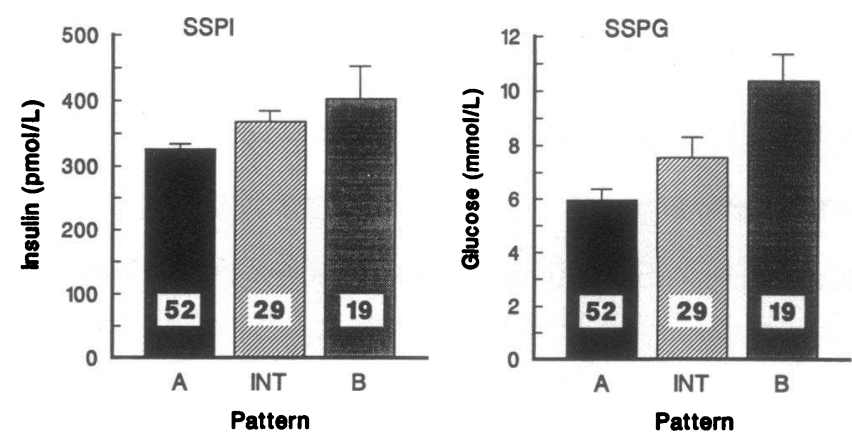

Figure 2. SSPI and SSPG concentrations at the end of a $180-\mathrm{min}$ infusion of somatostatin, insulin, and glucose. Subjects were divided into pattern A, an intermediate pattern (INT), and pattern B on the basis of their LDL particle diameter. The numbers in the bars represent the number of individuals in each group. 
Table II. Lipid and Lipoprotein Concentrations in Subjects Divided into Pattern A, an Intermediate Pattern, or Pattern B on the Basis of their LDL Particle Diameter*

\begin{tabular}{|c|c|c|c|}
\hline Variable & $\begin{array}{c}\mathrm{A} \\
(n=52)\end{array}$ & $\begin{array}{l}\text { INT } \\
(n=29)\end{array}$ & $\begin{array}{c}\text { B } \\
(n=19)\end{array}$ \\
\hline & \multicolumn{3}{|c|}{$\mathrm{mmol} / \mathrm{liter}$} \\
\hline TG & $0.77 \pm 0.05$ & $1.33 \pm 0.17^{\ddagger}$ & $1.98 \pm 0.15^{511}$ \\
\hline VLDL-TG & $0.43 \pm 0.04$ & $0.92 \pm 0.16^{\ddagger}$ & $1.51 \pm 0.15^{\S 11}$ \\
\hline IDL-TG & $0.10 \pm 0.006$ & $0.11 \pm 0.008$ & $0.13 \pm 0.006^{\S}$ \\
\hline LDL-TG & $0.15 \pm 0.006$ & $0.18 \pm 0.011$ & $0.22 \pm 0.017^{\S \mid}$ \\
\hline HDL-TG & $0.09 \pm 0.004$ & $0.12 \pm 0.008^{\ddagger}$ & $0.12 \pm 0.007^{\S}$ \\
\hline Cholesterol & $4.65 \pm 0.10$ & $4.80 \pm 0.18$ & $5.68 \pm 0.22^{5 \|}$ \\
\hline VLDL-cholesterol & $0.41 \pm 0.04$ & $0.73 \pm 0.12$ & $1.15 \pm 0.14^{\S \|}$ \\
\hline IDL-cholesterol & $0.23 \pm 0.02$ & $0.26 \pm 0.03$ & $0.44 \pm 0.04^{\S \|}$ \\
\hline LDL-cholesterol & $2.52 \pm 0.07$ & $2.60 \pm 0.12$ & $2.95 \pm 0.18^{\S}$ \\
\hline HDL-cholesterol & $1.45 \pm 0.05$ & $1.34 \pm 0.05$ & $1.12 \pm 0.06^{\S}$ \\
\hline $\begin{array}{l}\text { Cholesterol/HDL- } \\
\text { cholesterol }\end{array}$ & $3.40 \pm 0.15$ & $3.76 \pm 0.20$ & $5.50 \pm 0.50^{8 \| 11}$ \\
\hline
\end{tabular}

${ }^{*}$ Mean \pm SE. INT, intermediate. ${ }^{\ddagger}$ INT pattern significantly different $(P<0.05)$ from pattern A. ${ }^{\S}$ Pattern B significantly different $(P$ $<0.05)$ from pattern A. "Pattern B significantly different $(P<0.05)$ from INT pattern.

tern ( $7.5 \pm 0.7 \mathrm{mmol} /$ liter). Since the SSPI values (Fig. 2, left) were also higher in those with pattern $B(414 \pm 55 \mathrm{pmol} /$ liter $)$ than in individuals with either pattern $A(338 \pm 13 \mathrm{pmol} /$ liter $)$ or the intermediate pattern $(380 \pm 25 \mathrm{pmol} /$ liter $)$; subjects with pattern B were insulin resistant compared with the other two groups. It should be noted that SSPG values were also significantly higher than normal $(P<0.05)$ in those with the intermediate pattern.

Table II displays lipid and lipoprotein concentrations for the three groups, and these results indicate that subjects with pattern B had significantly different values for all variables measured than did those with pattern A. Furthermore, significant differences existed between those with the intermediate and $B$ patterns for most of the variables measured.

To define the relationships between LDL particle size and the other variables measured, the diameter of the predominant LDL gradient gel peak for each subject was used. Direct correlation coefficients between LDL diameter and the experimental variables measured are shown in Table III. It is apparent that the older and the greater the BMI of an individual, the smaller the LDL diameter. Males had significantly smaller LDL particles, and blood pressure also correlated negatively with LDL diameter, both systolic and diastolic. LDL diameter also correlated with the measures of glucose and insulin metabolism; the smaller the LDL diameter, the greater the plasma glucose and insulin responses to the oral glucose load. This indirect evidence of a relationship between LDL diameter and insulin resistance was supported by the significant correlation between SSPG and LDL diameter. Finally, all measures of plasma lipid and lipoprotein concentration were significantly correlated with LDL diameter. Partial correlation coefficients, taking differences in BMI, age, and gender into consideration, are also seen in Table III and demonstrate that all of the rela- tionships with LDL diameter remain significant, with the exception of LDL-cholesterol concentration.

Previous studies have demonstrated a strong relationship between SSPG (insulin resistance) and both the plasma insulin response to oral glucose and the fasting TG concentration, and this was true of the current data (SSPG vs. insulin response, $r=$ $0.60, P<0.001$ and SSPG vs. TG, $r=0.65, P<0.001$, Spearman correlation). Since both of these variables also correlated significantly with LDL particle diameter, partial correlation coefficients were calculated to see if the relationship noted between SSPG and LDL particle size was independent of the insulin response and / or the TG level. When the partial correlation between SSPG and LDL particle diameter was determined, taking into account the TG concentration, the simple correlation between SSPG and LDL particle diameter decreased from -0.44 to -0.04 and was no longer significant. The correlation coefficient between SSPG and LDL particle diameter also fell $(-0.44$ to -0.28$)$ when the insulin response was taken into account, but in this instance the partial correlation between the two variables remained significant ( $P$ $<0.006$ ).

To further evaluate the relationship between LDL particle diameter and insulin resistance and plasma TG concentration, multiple regression analysis was performed with age, BMI, gender, SSPG, and TG as the independent variables. When this was done, only plasma TG concentration emerged as an independent predictor of LDL particle diameter $(P<0.001)$. The addition of insulin response to the above model had no impact on the independent relationship between TG and LDL particle diameter. However, when cholesterol concentrations in the plasma and lipoprotein fractions were added to the model (excluding VLDL cholesterol because of its close correlation with

Table III. Simple and Partial Correlation Coefficients between LDL Diameter and Other Variables Measured

\begin{tabular}{|c|c|c|c|c|}
\hline \multirow[b]{2}{*}{ Variable } & \multicolumn{2}{|c|}{ Simple } & \multicolumn{2}{|c|}{ Partial } \\
\hline & $r$ & $P$ & $r$ & $P$ \\
\hline Age $(y r)$ & -0.21 & -0.04 & - & - \\
\hline $\operatorname{BMI}\left(\mathrm{kg} / \mathrm{m}^{2}\right)$ & -0.33 & -0.0007 & - & - \\
\hline Gender (male/female) & -0.25 & 0.02 & - & - \\
\hline Systolic blood pressure $(\mathrm{mmHg})$ & -0.34 & 0.0005 & -0.21 & 0.04 \\
\hline Diastolic blood pressure $(\mathrm{mmHg})$ & -0.34 & 0.0006 & -0.22 & 0.03 \\
\hline Glucose area $(m m o l / l i t e r \cdot h)$ & -0.41 & 0.0001 & -0.32 & 0.002 \\
\hline Insulin area $(p m o l / l i t e r \cdot h)$ & -0.38 & 0.0001 & -0.26 & 0.01 \\
\hline SSPG (mmol/liter) & -0.44 & 0.0001 & -0.39 & 0.0002 \\
\hline TG (mmol/liter) & -0.65 & 0.0001 & -0.56 & 0.0001 \\
\hline VLDL-TG (mmol/liter) & -0.63 & 0.0001 & -0.53 & 0.0001 \\
\hline IDL-TG (mmol/liter) & -0.38 & 0.0001 & -0.36 & 0.0003 \\
\hline LDL-TG (mmol/liter) & -0.37 & 0.0002 & -0.35 & 0.0004 \\
\hline HDL-TG (mmol/liter) & -0.35 & 0.0004 & -0.36 & 0.0003 \\
\hline Cholesterol (mmol/liter) & -0.40 & 0.0001 & -0.31 & 0.002 \\
\hline VLDL-Chol (mmol/liter) & -0.55 & 0.0001 & -0.45 & 0.0001 \\
\hline IDL-Chol (mmol/liter) & -0.40 & 0.0001 & -0.36 & 0.0004 \\
\hline LDL-Chol (mmol/liter) & -0.22 & 0.03 & -0.16 & NS \\
\hline HDL-Chol (mmol/liter) & 0.42 & 0.0001 & 0.33 & 0.001 \\
\hline
\end{tabular}


Table IV. Baseline Characteristics of Subjects Divided into Pattern A, an Intermediate Pattern, or Pattern B on the Basis of Their LDL Particle Diameter Matched for Age, BMI, and Gender*

\begin{tabular}{lccc}
\hline \multicolumn{1}{c}{ Variable } & $\begin{array}{c}\text { A } \\
(n=19)\end{array}$ & $\begin{array}{c}\text { INT } \\
(n=17)\end{array}$ & $\begin{array}{c}\text { B } \\
(n=19)\end{array}$ \\
\hline Age $(y r)$ & $42 \pm 13$ & $51 \pm 12^{\ddagger}$ & $45 \pm 11$ \\
BMI $\left(k g / m^{2}\right)$ & $26.0 \pm 3.5$ & $25.9 \pm 3.2$ & $26.5 \pm 3.9$ \\
Males/female & $12 / 7$ & $10 / 7$ & $12 / 7$ \\
LDL diameter $(\dot{A})$ & $266 \pm 2$ & $261 \pm 4^{\ddagger}$ & $250 \pm 4^{\text {\&॥ }}$ \\
Systolic blood pressure $(\mathrm{mmHg})$ & $113 \pm 13$ & $120 \pm 15$ & $127 \pm 18^{\S}$ \\
Diastolic blood pressure $(\mathrm{mmHg})$ & $73 \pm 8$ & $72 \pm 10$ & $81 \pm 10^{\text {s॥ }}$
\end{tabular}

* Mean \pm SD. INT, intermediate. ${ }^{\ddagger}$ INT pattern significantly different $(P<0.05)$ from pattern A. ${ }^{8}$ Pattern B significantly different $(P$ $<0.05)$ from pattern A. "Pattern B significantly different $(P<0.05)$ from INT pattern.

TG), significant independent relationships were demonstrated between LDL particle diameter and both TG $(P<0.005)$ and intermediate density lipoprotein (IDL) cholesterol $(P<0.02)$.

The partial correlation coefficients shown in Table III attest to the relationship between LDL particle diameter and the other variables measured after statistically adjusting for age, BMI, and gender. To pursue this issue further, three groups were matched in regards to these variables. The characteristics of these three groups are shown in Table IV, demonstrating their similarity in terms of age, BMI, and gender distribution. As before, the LDL particle size was smaller and blood pressure was higher in pattern B subjects.

The results of the glucose tolerance tests of the three matched groups are shown in Fig. 3, and this subset of pattern $B$ individuals was also glucose intolerant and hyperinsulinemic compared with the other two groups $(P=0.05-0.005)$. Finally, the results in Fig. 4 demonstrate that SSPG values were higher in group B individuals then in the other groups, despite their higher SSPI concentrations, i.e., they are insulin resistant.

Lipid and lipoprotein concentrations of the matched groups are listed in Table $\mathrm{V}$ and indicate that the dyslipidemia of subjects with pattern B persists when they are closely matched with the other two groups.
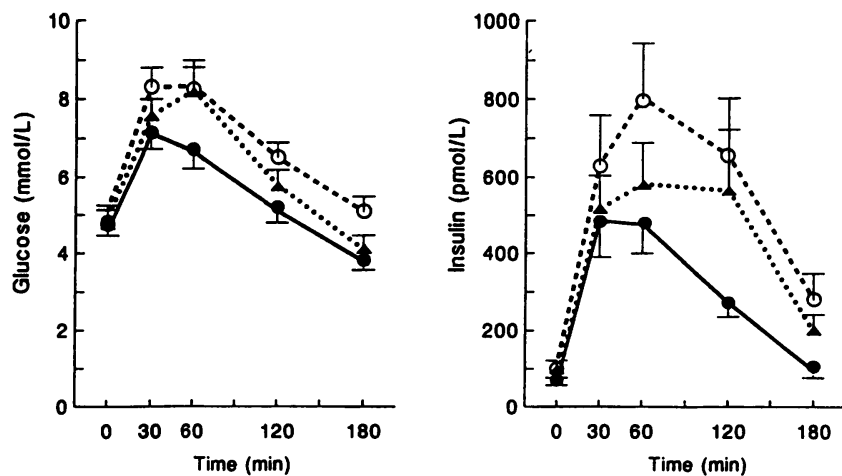

Figure 3. Plasma glucose (left) and insulin (right) responses to a 75-g oral glucose challenge in subjects divides into pattern $A(\bullet-\bullet)$, an intermediate pattern $(\Delta \cdots \Delta)$, and pattern $B(0--0)$ on the basis of their LDL particle diameter, and then matched for age, gender, and BMI.
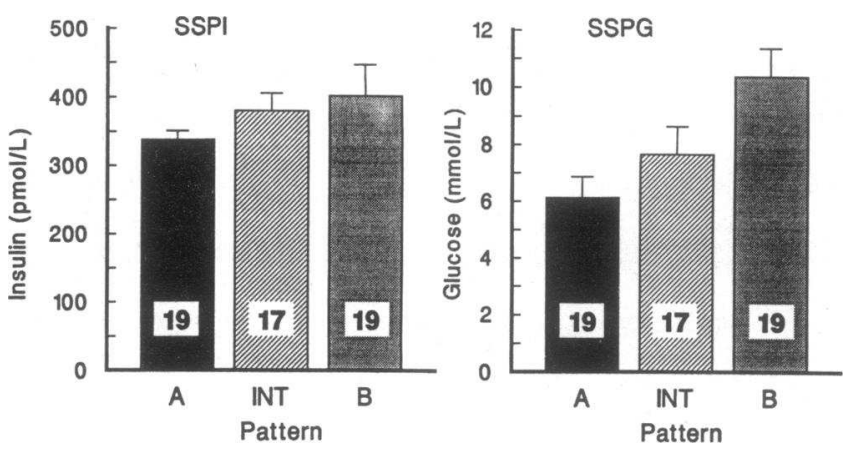

Figure 4. SSPI and glucose SSPG concentration at the end of a 180min infusion of somatostatin, insulin, and glucose. Subjects were divided into pattern $\mathrm{A}$, an intermediate pattern $(I N T)$, or pattern B on the basis of their LDL particle diameter, and then matched for age, gender, and BMI. The numbers in the bars represent the number of individuals in each group.

\section{Discussion}

The goal of this study was to see if individuals who were classified as pattern B on the basis of their LDL particle diameter were also insulin resistant. The results shown in Figs. 2 and 4 indicate that this was the case, and the relationship persisted when differences in gender, age, and degree of obesity were taken into consideration. In support of this conclusion was the observation (Figs. 1 and 3) that individuals with the pattern B subclass were also glucose intolerant and hyperinsulinemic in comparison to the other two groups.

In addition to insulin resistance, glucose intolerance, and hyperinsulinemia, pattern B individuals were also dyslipidemic, with higher plasma TG and lower HDL-cholesterol con-

Table V. Lipid and Lipoprotein Concentrations in Subjects Divided into Pattern A, an Intermediate Pattern, or Pattern $B$ on the Basis of Their LDL Particle Diameter Matched for Age, BMI, and Gender*

\begin{tabular}{|c|c|c|c|}
\hline Variable & $\begin{array}{c}\mathrm{A} \\
(n=19)\end{array}$ & $\begin{array}{c}\text { INT } \\
(n=17)\end{array}$ & $\begin{array}{c}\text { B } \\
(n=19)\end{array}$ \\
\hline & \multicolumn{3}{|c|}{$\mathrm{mmol} / \mathrm{liter}$} \\
\hline TG & $0.81 \pm 0.09$ & $1.52 \pm 0.26^{\ddagger}$ & $1.98 \pm 0.15^{\S}$ \\
\hline VLDL-TG & $0.45 \pm 0.08$ & $1.10 \pm 0.25^{\ddagger}$ & $1.51 \pm 0.15^{8}$ \\
\hline IDL-TG & $0.10 \pm 0.01$ & $0.11 \pm 0.01$ & $0.13 \pm 0.01$ \\
\hline LDL-TG & $0.16 \pm 0.01$ & $0.18 \pm 0.01$ & $0.22 \pm 0.02^{\text {sU }}$ \\
\hline HDL-TG & $0.09 \pm 0.01$ & $0.13 \pm 0.01^{\ddagger}$ & $0.12 \pm 0.01^{8}$ \\
\hline Cholesterol & $4.64 \pm 0.15$ & $4.97 \pm 0.18$ & $5.68 \pm 0.22^{811}$ \\
\hline VLDL-cholesterol & $0.49 \pm 0.08$ & $0.82 \pm 0.16$ & $1.15 \pm 0.14^{8}$ \\
\hline IDL-cholesterol & $0.20 \pm 0.03$ & $0.25 \pm 0.04$ & $0.44 \pm 0.44^{8 \text { ॥ }}$ \\
\hline LDL-cholesterol & $2.49 \pm 0.12$ & $2.62 \pm 0.12$ & $2.95 \pm 0.18^{8}$ \\
\hline HDL-cholesterol & $1.36 \pm 0.08$ & $1.27 \pm 0.06$ & $1.12 \pm 0.06^{8}$ \\
\hline Cholesterol/HDL-cholesterol & $3.63 \pm 0.28$ & $4.03 \pm 0.23$ & $5.50 \pm 0.50^{5 \mathrm{1} 11}$ \\
\hline
\end{tabular}

${ }^{*}$ Mean \pm SE. INT, intermediate. ${ }^{\ddagger}$ INT pattern significantly different $(P<0.05)$ from pattern A. ${ }^{5}$ Pattern B significantly different $(P$ $<0.05)$ from pattern A. "Pattern B significantly different $(P<0.05)$ from INT pattern. 
centrations and higher systolic and diastolic blood pressures. Further, these relationships all persisted when differences in gender distribution, age, and obesity were taken into consideration. Thus, although the specific goal of this study was to define the relationship if any between LDL particle diameter and glucose and insulin metabolism, it seems necessary to also comment in a somewhat broader fashion on the multiple relationships that have been described. Several years ago it was suggested that a cluster of abnormalities, including glucose intolerance, increased blood pressure, high plasma TG concentrations, and low HDL-cholesterol concentrations, were associated with insulin resistance and/or hyperinsulinemia (23). At that time, it was suggested that the cluster of changes noted above constitute a syndrome (X). Since then, several reports have been published that have demonstrated the clustering of similar changes $(24,25)$. More recently, it has been shown that increases in serum uric acid concentration and plasminogen activator inhibitor-1 also seem to be associated with insulin resistance and/or hyperinsulinemia, hypertriglyceridemia, high blood pressure, and a low HDL cholesterol (26-29). On the basis of the data presented in this study, there is now strong substantial evidence that smaller and denser LDL particles be added to the group of changes initially described under the rubric of Syndrome X (23).

Although there appears to be some general agreement as to the changes that constitute Syndrome $X$, issues remain as to the nature of the relationship between the variables that make up the cluster. For example, when initially defined, it was suggested that insulin resistance was the basic defect and the other events noted, e.g., hypertriglyceridemia, were secondary. However, it has also been argued that hypertriglyceridemia can cause insulin resistance (30). At the present time, the information necessary to decide between these two alternatives does not seem to be available. However, the current data provide evidence that any relationship that exists between insulin resistance and LDL particle diameter is likely to be exerted via its impact on lipoprotein metabolism. Significantly, when multiple regression analysis was performed, only plasma TG and/or IDL-cholesterol concentrations were shown to be independent predictors of LDL particle diameter. This latter observation is of additional interest given demonstration of the relationship between IDL and progression of coronary heart disease (31).

In conclusion, individuals with small, dense LDL particles (pattern B) are relatively insulin resistant, glucose intolerant, hyperinsulinemic, hypertensive, and have a lower HDL-cholesterol concentration. These observations support the view that this change in LDL composition be added to the cluster of abnormalities previously referred to as Syndrome X (23). Recent studies in normal (32) and hyperlipidemic (33) families have indicated that LDL subclass patterns are influenced by a major gene. Its linkage to a locus near the LDL and insulin receptor genes on chromosomes $19 p$ has been demonstrated (34). The present finding indicates that the allele responsible for expression of LDL subclass pattern B may also predispose to insulin resistance.

\section{Acknowledgments}

We thank Patricia Blanche, Laura Hall, Bahareh Sahami, Tammy Wang, and Mo-Oi Chang for laboratory assistance. Our special thanks goes to Yi Nina Chen who performed all the statistical analysis. We also thank Joyce Loewy for the preparation of the manuscript.
This work was supported by National Institutes of Health research grants (RR 00070 and HL 08506, Program Project HL 18574) and by a grant from the National Dairy Promotion and Research Board administered in cooperation with a the National Dairy Council. This work was conducted at Lawrence Berkeley Laboratory through the U.S. Department of Energy under Contract \#DE-AC03-76SF00098 and Stanford University Medical Center, Stanford, CA.

\section{References}

1. Fisher, W. R., M. G. Hammond, and G. L. Warmke. 1972. Measurements of the molecular weight variability of plasma low density lipoproteins among normals and subjects with hyperbetalipoproteinemia. Demonstration of macromolecular heterogeneity. Biochemistry. 11:519-525.

2. Krauss, R. M., and D. J. Burke. 1982. Identification of multiple subclasses of plasma low density lipoproteins in normal humans. J. Lipid Res. 23:97-104.

3. Kraemer, F. B., Y.-D. I. Chen, R. M. C. Cheung, and G. M. Reaven. 1982. Are the binding and degradations of low density lipoprotein altered in Type 2 (non-insulin-dependent) diabetes mellitus? Diabetologia. 23:28-33.

4. Crouse, J. R., J. S. Parks, and H. M. Schey. 1985. Studies of low density lipoprotein molecular weight in human beings with coronary artery disease. $J$. Lipid Res. 26:566-574.

5. Austin, M. A., J. L. Breslow, C. H. Hennekens, J. E. Buring, W. S. Willett, and R. M. Krauss. 1988. Low-density lipoprotein subclass patterns and risk of myocardial infarction. J. Am. Med. Assoc. 260:1917-1921.

6. Austin, M. A., M.-C. King, K. M. Vranizan, and R. M. Krauss. 1990. Atherogenic lipoprotein phenotype: a proposed genetic marker for coronary heart disease risk. Circulation. 82:495-506.

7. Reaven, G. M., R. L. Lerner, M. P. Stern, and J. W. Farquhar. 1967. Role of insulin in endogenous hypertriglyceridemia. J. Clin. Invest. 46:1756-1767.

8. Olefsky, J. M., J. W. Farquhar, and G. M. Reaven. 1974. Reappraisal of the role of insulin in hypertriglyceridemia. Am. J. Med. 57:551-560.

9. Zavaroni, I., S. Mazza, L. Luchetti, G. Buonanno, P. A. Bonati, M. Bergonazani, M. Passeri, and G. M. Reaven. 1990. High plasma insulin and triglyceride concentrations and blood pressure in offspring of people with impaired glucose tolerance. Diabetic Med. 7:494-498.

10. Stalder, M., D. Pometta, and A. Suenram. 1981. Relationship between plasma insulin levels and high density lipoprotein cholesterol levels in healthy men. Diabetologia. 21:544.

11. Laws, A., and G. M. Reaven. 1992. Evidence for an independent relationship between insulin resistance and fasting plasma HDL-cholesterol, triglyceride and insulin concentrations. J. Intern. Med. 231:25-30.

12. Wahlefeld, A. W. 1974. Triglycerides. Determination after enzymatic hydrolysis. In Methods of Enzymatic Analysis. H. U. Bergmeyer, editor. Academic Press, New York. 1831-1835.

13. Allain, C. A., L. S. Poon, C. S. G. Chun, W. Richmond, and P. C. Fu. 1974. Enzymatic determination of total serum cholesterol. Clin. Chem. 20:470475.

14. Swislocki, A. L. M., B. B. Hoffman, W. H.-H. Sheu, Y.-D. I. Chen, and G. M. Reaven. 1989. Effect of prazosin treatment on carbohydrate and lipoprotein metabolism in patients with hypertension. Am. J. Med. 86(Suppl. 1B):14 18.

15. Kadish, A. H., R. H. Litle, and J. C. Sternberg. 1968. A new and rapid method for determination of glucose by measurement of rate of oxygen consumption. Clin. Chem. 14:116-131.

16. Hales, C. N., and P. J. Randle. 1963. Immunoassay of insulin with insulinantibody precipitate. Biochem. J. 88:137-146.

17. Shen, D.-C., S.-M. Shieh, M.-T. Fuh, D.-A. Wu, Y.-D. I. Chen, and G. M. Reaven. 1988. Resistance to insulin-stimulated-glucose uptake in patients with hypertension. J. Clin. Endocrinol. Metab. 66:580-583.

18. Shen, S.-W., G. M. Reaven, and J. Farquhar. 1970. Comparison of impedence to insulin-mediated glucose uptake in normal subjects and in subjects with latent diabetes. J. Clin. Invest. 49:2151-2160.

19. Krauss, R. M., and D. J. Burke. 1982. Identification of multiple subclasses of plasma low density lipoproteins in normal humans. J. Lipid Res. 28:97-104.

20. Winer, B. J. 1972. Design and analysis of factorial experiments: nested factors (hierarch designs). In Statistical Principles in Experimental Design. B. J. Winer, editor. McGraw-Hill, New York. 359-366.

21. Kendall, M. G., and A. Stuart. 1961. The Advanced Theory of Statistics. Vol. 2. Griffin, London, England. 317-345.

22. Snedecor, G. W., and W. G. Cochran. 1980. Statistical Methods. 7th ed. The Iowa State University Press, Ames, IA. 507 pp.

23. Reaven, G. M. 1988. Role of insulin resistance in human disease. Diabetes. 37:1595-1607.

24. Haffner, S. M. 1990. Cardiovascular risk factor in confirmed prediabetic individuals: does the clock for coronary heart disease start ticking before the onset of clinical diabetes? J. Am. Med. Assoc. 263:2893-2898. 
25. Ferrannini, E., S. M. Haffner, B. D. Mitchell, and M. P. Stern. 1991. Hyperinsulinaemia: the key feature of a cardiovascular and metabolic syndrome. Diabetologia. 3:416-422.

26. Facchini, F., Y.-D. I. Chen, C. B. Hollenbeck, and G. M. Reaven. 1991 Relationship between resistance to insulin-medited glucose uptake, urinary uric acid clearance, and plasma uric acid concentration. J. Am. Med. Assoc. 266:3008-3011.

27. Landin, K., L. Tengborn, and U. Smith. 1990. Elevated fibrinogen and plasminogen activator (PAI-1) in hypertension are related to metabolic risk factors for cardiovascular disease. J. Intern. Med. 227:273-278.

28. Juhan-Vague, I., C. Roul, M. D. Alessi, J. P. Ardissone, M. Heim, and P. Vague. 1989. Increased plasminogen activator inhibitor activity in non-insulindependent diabetic patients. Relationship with plasma insulin. Thromb. Haemostasis. 61:370-373.

29. Juhan-Vague, I., M. C. Alessi, and P. Vague. 1991. Increased plasma plasminogen activator inhibitor 1 levels. A possible link between insulin resistance and atherothrombosis. Diabetologia. 34:457-462.
30. Steiner, G. 1991. Altering triglyceride concentrations changes insulin-glucose relationships in hypertriglyceridemic patients: double-blind study with gemfibrozil with implications for atherosclerosis. Diabetes Care. 14:1077-1078.

31. Krauss, RM., F. T. Lindgren, P. T. Williams, S. F. Kelsey, J. Brensike, K. Vranizan, K. M. Detre, and R. I. Levy. 1987. Intermediate density lipoproeins and coronary artery disease progression in hypercholesterolemic men. Lance ii:62-66.

32. Austin, M., M. C. King, K. M. Vranizan, B. Newman, and R. M. Krauss. 1988. Inheritance of low-density lipoprotein subclass patterns: results of complex segregation analysis. Am. J. Hum. Genet. 43:838-846.

33. Austin, M. A., J. D. Brunzell, W. I. Fitch, and R. M. Krauss. 1990 Inheritance of low density lipoprotein subclass patterns in familial combined hyperlipidemia. Arteriosclerosis. 10:520-530.

34. Nishina, P. M., J. P. Johnson, J. K. Naggert, and R. M. Krauss. 1992 Linkage of atherogenic lipoprotein phenotype to the low density lipoprotein receptor locus on the short arm of chromosome 19. Proc. Natl. Acad. Sci. USA 89:708-712. 